\title{
A Substitute of Harmonic Majorization
}

\section{Liliana De Rosa And Carlos Segovia}

\begin{abstract}
A substitute of harmonic majorization of powers of harmonic functions in $\mathbb{R}_{+}^{2}$ is given for one sided kernels and one sided doubling weights. The substitute of harmonic majorization obtained is applied to the study of a generalization of the $T_{\lambda, r}$ functions of Fefferman and Stein.
\end{abstract}

It is well known, see [SW] and [GR], that if $u(x, t)$ is a harmonic function defined on the upper half plane $(t>0), 1 \leq q<\infty$ and $\delta>0$, then there exists a function $g(x) \geq 0$ such that

$$
|u(x, t)|^{\delta} \leq P_{t} * g(x),
$$

where $P_{t}(x)=(1 / \pi)\left(t /\left(t^{2}+x^{2}\right)\right)$ is the Poisson kernel on $\mathbb{R}_{+}^{2}$ and

$$
\int g(x)^{q} d x \leq \int u^{*}(x)^{q \delta} d x
$$

where $u^{*}(x)=\sup _{|x-y|<t}|u(y, t)|$. The purpose of this paper is to generalize these results to kernels different from the Poisson kernel and other maximal functions. The result we obtain is stated in Theorem (5). We apply Theorem (5) to the study of a one sided version of the $T_{\lambda, r}$ function of Fefferman and Stein $[\mathrm{FS}]$ and the results obtained in this direction are stated in Theorem (18).

The Lebesgue measure of a set $E \subseteq \mathbb{R}$ is denoted by $|E|$ and its characteristic function by $\chi_{E}(x)$. As usual, a weight $w(x)$ is a Lebesgue measurable and non-negative function defined on $\mathbb{R}$. A function $f(x)$ belongs to $L^{p}(w), 0<p \leq \infty$, if $\|f\|_{L^{p}(w)}=\left(\int_{-\infty}^{\infty}|f(x)|^{p} w(x) d x\right)^{1 / p}$ is finite. If $w(x) \equiv 1$, we simply write $\|f\|_{L^{p}}$ or $\|f\|_{p}$. If $E \subseteq \mathbb{R}$ is a Lebesgue measurable set, we denote its $w$-measure by $w(E)=\int_{E} w(t) d t$. The set function $w(E)$ is a measure on the $\sigma$-algebra of all Lebesgue measurable subset of $\mathbb{R}$. If 
$w([x, x+2 t]) \leq c_{w} \cdot w([x, x+t])$ holds for every $x \in \mathbb{R}$ and $t>0$, we shall say that $w(x)$ is a right hand doubling weight.

A weight $w(x)$ belongs to the class $A_{p}^{-}, 1<p<\infty$, if there exists a constant $c_{w, p}$ such that for every $a<b<c$, we have

$$
\left(\int_{b}^{c} w(y) d y\right)\left(\int_{a}^{b} w(y)^{-1 /(p-1)} d y\right)^{p-1} \leq c_{w, p}(c-a)^{p}
$$

and $w(x)$ belongs to $A_{1}^{-}$if

$$
\sup _{s>0} \frac{1}{s} \int_{x}^{x+s} w(y) d y \leq c_{w, 1} w(x)
$$

holds for almost every $x$ in $\mathbb{R}$.

We define the left sided Hardy-Littlewood maximal $M^{-} f(x)$ as

$$
M^{-} f(x)=\sup _{s>0} \frac{1}{s} \int_{x-s}^{x}|f(y)| d y .
$$

E. Sawyer proved in $[\mathrm{S}]$ that in the case $1<p<\infty$, there exists a constant $c_{w, p}$ such that

$$
\left\|M^{-} f\right\|_{L^{p}(w)} \leq c_{w, p}\|f\|_{L^{p}(w)}
$$

if and only if $w \in A_{p}^{-}$. Moreover, the inequality

$$
s w\left(\left\{x: M^{-} f(x)>s\right\}\right) \leq c_{w, 1}\|f\|_{L^{1}(w)}
$$

holds for every $s>0$, if and only if $w \in A_{1}^{-}$.

Let $w(x) \geq 0$ defined on $\mathbb{R}$. Given a Lebesgue measurable function $f(x)$, for every bounded interval $I$ we define $m_{I}(f, w)$ as

$$
m_{I}(f, w)=\frac{1}{w(I)} \int_{I}|f(y)| w(y) d y, \quad \text { if } 0<w(I)<\infty
$$

and

$$
m_{I}(f, w)=0, \quad \text { if } w(I)=0 \text { or } w(I)=\infty .
$$

Then, the maximal function $M_{w} f(x)$ is defined as

$$
M_{w} f(x)=\sup _{x \in I} m_{I}(f, w)
$$


where the supremum is taken over all bounded intervals $I$ such that $x \in I$. Since we work on the real line it is well known that there exists a constant $c_{q}$ such that the weighted norm inequality

$$
\left\|M_{w} f\right\|_{L^{q}(w)} \leq c_{q}\|f\|_{L^{q}(w)}
$$

holds if $1<q<\infty$, and

$$
s w\left(\left\{x \in \mathbb{R}: M_{w} f(x)>s\right\}\right) \leq c\|f\|_{L^{1}(w)}
$$

holds for every $s>0$.

As usual, by $C_{0}^{\infty}$ we mean the class of all functions with compact support and derivatives of all orders, and $\mathcal{S}$ stand for the class of functions with rapidly decreasing derivatives of all orders.

Let $\gamma$ be a non-negative integer and $\Phi_{\gamma}$ the class of all $C_{0}^{\infty}$-functions $\psi(x)$, such that there exists an interval $I=[0, \beta]$ satisfying

(i) support $(\psi) \subset I$

(ii) $|I|^{\gamma+1}\left\|D^{\gamma} \psi\right\|_{\infty} \leq 1$.

Given a bounded function $f(x)$ with compact support, we shall consider the left sided maximal function $f_{-, \gamma}^{*}$ defined as

$$
f_{-, \gamma}^{*}(x)=\sup |f * \psi(x)|
$$

where the supremum is taken over all $\psi$ belonging to $\Phi_{\gamma}$, see [RS].

Let $r>0$ and $s>1+r$, we define the function

$$
h(x)=\frac{x^{r}}{(1+x)^{s}} \chi_{(0, \infty)}(x)
$$

For any function $\varphi(x)$ we denote by $\varphi_{t}(x)$ the function $\varphi_{t}(x)=t^{-1} \varphi(x / t)$.

With these definitions and notations we can state the main result of this paper.

Theorem 5. Let $w(x)$ be a right hand doubling weight, $k$ a non-negative integer and $\varphi(x) \in \mathcal{S}$ with support contained in $[0, \infty)$. Given a bounded function $f(x)$ with bounded support there exists a non-negative function $g(x)$ such that

$$
\left|f * \varphi_{t}(x)\right| \leq c\left(g * h_{t}(x)\right)^{k+2}
$$


and

$$
\int g(x)^{q} w(x) d x \leq c \int f_{-, k+1}^{*}(x)^{q /(k+2)} w(x) d x
$$

hold with $1 \leq q<\infty$ and a constant $c$ depending on $k, \varphi, h$ and $q$.

In order to prove Theorem (5) we shall need some results.

Lemma 8. Let $w(x)$ be a right hand doubling weight. If $\left\{x_{k}\right\}$ is a sequence of real numbers and $\left\{r_{k}\right\}$ and $\left\{\lambda_{k}\right\}$ are sequences of positive numbers, then given $a>1$, there exists a constant $c_{q, a}(w)$ such that

$$
\left\|\sum \lambda_{k} \chi_{\left(x_{k}, x_{k}+a r_{k}\right)}\right\|_{L^{q}(w)} \leq c_{q, a}(w)\left\|\sum \lambda_{k} \chi_{\left(x_{k}, x_{k}+r_{k}\right)}\right\|_{L^{q}(w)}
$$

holds for $1 \leq q<\infty$. The constant $c$ depends on $w$ and $q$.

Proof. The proof of this Lemma for a doubling weight is sketched in Lemma 4, page 115 of [ST]. For the sake of completeness we give the proof here. First of all we observe that it is enough to prove this lemma if $w\left(\left(x_{k}, x_{k}+r_{k}\right)\right)<\infty$ for every $k$. Actually, we can assume that $0<w\left(\left(x_{k}, x_{k}+r_{k}\right)\right)<\infty, k \geq 1$, and since $w(x)$ is a right hand doubling weight it follows that

$$
0<w\left(\left(x_{k}, x_{k}+a r_{k}\right)\right)<\infty
$$

for every $k$. Let $g(x) \geq 0$ and $M_{w} g(x)$ the maximal function defined in (1). For $x_{k} \leq z \leq x_{k}+r_{k}$, we have

$$
\int_{x_{k}}^{x_{k}+a r_{k}} g(x) w(x) d x \leq w\left(x_{k}, x_{k}+a r_{k}\right) M_{w} g(z)
$$

Therefore, since $w(x)$ is a right hand doubling weight, we get

$$
\int_{x_{k}}^{x_{k}+a r_{k}} g(x) w(x) d x \leq c \int_{x_{k}}^{x_{k}+r_{k}} M_{w} g(z) w(z) d z
$$

for every $k$. Then

$$
\sum_{k} \lambda_{k} \int_{x_{k}}^{x_{k}+a r_{k}} g(x) w(x) d x \leq c \sum_{k} \lambda_{k} \int_{x_{k}}^{x_{k}+r_{k}} M_{w} g(z) w(z) d z
$$

which is equivalent to 


$$
\begin{aligned}
\int_{-\infty}^{\infty}\left(\sum_{k} \lambda_{k} \chi_{\left(x_{k}, x_{k}+a r_{k}\right)}(x)\right) g(x) w(x) d x \\
\leq c \int_{-\infty}^{\infty}\left(\sum_{k} \lambda_{k} \chi_{\left(x_{k}, x_{k}+r_{k}\right)}(z)\right) M_{w} g(z) w(z) d z
\end{aligned}
$$

Applying Hölder's inequality and (2), we have that the right hand side above is bounded by

$$
C\left\|\sum_{k} \lambda_{k} \chi_{\left(x_{k}, x_{k}+r_{k}\right)}\right\|_{L^{q}(w)}\|g\|_{L^{q^{\prime}}(w)}
$$

and the Lemma follows immediately with $c_{q, a}(w)=C$.

Lemma 9. Let $-\infty<\alpha<\beta<\infty$ and $I=(\alpha, \beta)$. Let $k$ be a nonnegative integer and $a(x)$ a function satisfying

(i) $\|a\|_{\infty}<\infty$,

(ii) support of a $(x)$ contained in $I$ and

(iii) if $\ell$ is a non-negative integer, $\ell \leq k$, then $\int x^{\ell} a(x) d x=0$.

Then, for $\varphi(x) \in \mathcal{S}$ with support contained in $[0, \infty)$ we have

(a) if $x \leq \alpha$ then $\varphi_{t} * a(x)=0$,

(b) if $x \geq \alpha$ then for any integer $n \geq 1$

$$
\left|\varphi_{t} * a(x)\right| \leq \frac{\|a\|_{\infty}\left\|D^{n} \varphi\right\|_{\infty}}{(n+1) !}\left(\frac{x-\alpha}{t}\right)^{n+1},
$$

and

$$
\left|\varphi_{t} * a(x)\right| \leq\|a\|_{\infty}\|\varphi\|_{1}
$$

(c) if $x \geq \beta+(\beta-\alpha)$ then for any non-negative integers $u$ and $v$

$$
\left|\varphi_{t} * a(x)\right| \leq c_{k, u, v, \varphi}\|a\|_{\infty}\left(\frac{\beta-\alpha}{t}\right)^{k+2} \frac{\left(x-\frac{\alpha}{t}\right)^{u}}{\left(1+\frac{x-\alpha}{t}\right)^{v}}
$$

Proof. By a change of variables, it is enough to prove the Lemma for the case $\alpha=0$. Let us show part a). It is evident since $\varphi_{t} * a(x)$ is the convolution of functions supported on $[0, \infty)$. Part b) (10): Since $x=0$ is a zero of $\varphi$ 
of infinite order then $\varphi(z)=z^{n} D^{n} \varphi(\theta z) / n$ ! with $0<\theta<1$, for any integer $n \geq 1$.

Thus, $|\varphi(z)| \leq\left\|D^{n} \varphi\right\|_{\infty}|z|^{n} / n$ !. Then, we have

$$
\left|\varphi_{t} * a(x)\right| \leq\|a\|_{\infty} \int_{0}^{\beta}\left|\varphi_{t}(x-y)\right| d y \leq\|a\|_{\infty} \int_{0}^{x / t}|\varphi(z)| d z .
$$

Therefore,

$$
\left|\varphi_{t} * a(x)\right| \leq \frac{\|a\|_{\infty}\left\|D^{n} \varphi\right\|_{\infty}}{(n+1) !}(x / t)^{n+1} .
$$

Part b) (11) is obvious. As for part c), taking into account (iii) we have

$$
\varphi_{t} * a(x)=t^{-1} \int\left[\varphi\left(\frac{x-y}{t}\right)-\sum_{\ell=0}^{k}\left(\frac{-y}{t}\right)^{\ell} \frac{1}{\ell !} D^{\ell} \varphi\left(\frac{x}{t}\right)\right] a(y) d y
$$

Since $\varphi(x) \in \mathcal{S}$ and the support of $\varphi(x)$ is contained in $[0, \infty)$, given any non-negative integers $u$ and $v$ we have

$$
\left|D^{k+1} \varphi(z)\right| \leq c_{k, u, v, \varphi} z^{u} /(1+z)^{v}
$$

for $z \geq 0$. Then, by Taylor's formula the integrand in (12) is bounded by

$$
c_{k, u, v, \varphi} \frac{1}{(k+1) !}\|a\|_{\infty}\left(\frac{\beta}{t}\right)^{k+1} \frac{(x / t)^{u}}{(1+x / t)^{v}}
$$

thus, integrating we get c).

Lemma 13. Let $h(x)$ be the function defined in (4). Let $I=[\alpha, \beta]$ and $\widetilde{I}=[\alpha, \beta+\beta-\alpha]$. Then,

(a) if $x \leq \alpha$ then $h_{t} * \chi_{\widetilde{I}}(x)=0$,

(b) if $\alpha \leq x \leq \beta+\beta-\alpha$ and $x-\alpha \leq 2 t$ then

$$
h_{t} * \chi_{\widetilde{I}}(x) \geq \frac{3^{-s}}{r+1}\left(\frac{x-\alpha}{t}\right)^{r+1},
$$

(c) if $\alpha \leq x \leq \beta+\beta-\alpha$ and $x-\alpha \geq 2 t$ then

$$
h_{t} * \chi_{\widetilde{I}}(x) \geq 3^{-s}
$$

and 
(d) if $\beta+\beta-\alpha \leq x$ then

$$
h_{t} * \chi_{\widetilde{I}}(x) \geq 2^{-r} \frac{\beta-\alpha}{t} \frac{\left(\frac{x-\alpha}{t}\right)^{r}}{\left(1+\frac{x-\alpha}{t}\right)^{s}} .
$$

Proof. Without loss of generality we can assume that $\alpha=0$. Part (a) is obvious. If $0 \leq x \leq 2 \beta$ and $x \leq 2 t$, we have

$$
\begin{aligned}
h_{t} * \chi_{\widetilde{I}}(x) & =\int_{0}^{2 \beta} h_{t}(x-y) d y=\int_{0}^{x / t} h(z) d z \\
& =\int_{0}^{x / t} \frac{z^{r}}{(1+z)^{s}} d z \geq \frac{3^{-s}}{r+1}\left(\frac{x}{t}\right)^{r+1}
\end{aligned}
$$

which proves (b). Let $0 \leq x \leq 2 \beta$ and $x \geq 2 t$. We have

$$
h_{t} * \chi_{\widetilde{I}}(x)=\int_{0}^{x / t} h(z) d z \geq \int_{1}^{2} \frac{z^{r}}{(1+z)^{s}} d z \geq 3^{-s}
$$

which proves (c). Finally, if $x \geq 2 \beta$,

$$
\begin{aligned}
h_{t} * \chi_{\widetilde{I}}(x) & =\int_{0}^{2 \beta} h_{t}(x-y) d y \geq \int_{0}^{\beta} h_{t}(x-y) d y \\
& =\int_{x-\beta / t}^{x / t} h(z) d z \geq \frac{\beta}{t} \frac{\left(\frac{x-\beta}{t}\right)^{r}}{(1+x / t)^{s}} \geq 2^{-r} \frac{\beta}{t} \frac{(x / t)^{r}}{(1+x / t)^{s}},
\end{aligned}
$$

which proves part (d).

From Lemmas 9 and 13 we get the following proposition:

Proposition 14. Let $I, a(x)$ and $\varphi(x)$ as in Lemma 9 and, $h(x)$ and $\widetilde{I}$, as in Lemma 13. If $u=r(k+2), v=s(k+2)$ and $n=(r+1)(k+2)-1$, we have

$$
\left|\varphi_{t} * a(x)\right| \leq c_{r, s, k, \varphi}\|a\|_{\infty}\left[h_{t} * \chi_{\widetilde{I}}(x)\right]^{k+2}
$$


Proof. It is obvious that (15) holds if $x \leq \alpha$. Let $\alpha \leq x \leq \beta+\beta-\alpha$ and $x-\alpha \leq 2 t$. By (10) and part b) of Lemma (13), we have

$$
\left|\varphi_{t} * a(x)\right| \leq \frac{(r+1)^{k+2}}{(n+1) !} 3^{s(k+2)}\left\|D^{n+1} \varphi\right\|_{\infty}\|a\|_{\infty}\left[h_{t} * \chi_{\widetilde{I}}(x)\right]^{k+2}
$$

and therefore (15) holds in this case. If $\alpha \leq x \leq \beta+\beta-\alpha$ and $x-\alpha \geq 2 t$, by (11) and part (c) of Lemma (13) it follows that

$$
\left|\varphi_{t} * a(x)\right| \leq 3^{s(k+2)}\|\varphi\|_{1}\|a\|_{\infty}\left[h_{t} * \chi_{\widetilde{I}}(x)\right]^{k+2},
$$

proving (15) also in this case. Finally if $x \geq \beta+\beta-\alpha$ by part (c) of Lemma (9) and part (d) of Lemma (13), we get

$$
\begin{aligned}
\left|\varphi_{t} * a(x)\right| & \leq c_{k, u, v, \varphi}\|a\|_{\infty}\left(\frac{\beta-\alpha}{t}\right)^{k+2} \frac{((x-\alpha) / t)^{u}}{(1+(x-\alpha) / t)^{v}} \\
& \leq c_{k, r, s, \varphi} 2^{r(k+2)}\|a\|_{\infty}\left[h_{t} * \chi_{\widetilde{I}}(x)\right]^{k+2}
\end{aligned}
$$

ending the proof.

Let $f(x)$ be a bounded function with bounded support and let $f_{-, k+1}^{*}(x)$ be the maximal function defined in (3). This function is finite everywhere on $\mathbb{R}$. The sets $\Omega_{i}=\left\{x: f_{-, k+1}^{*}(x)>2^{i}\right\}, i \in \mathbb{Z}$, are open and bounded. By Theorem (2.2) of [RS], if $I_{i j}$ stand for the connected components of $\Omega_{i}$, there exist functions $a_{i j}(x)$ such that

(i) $\left\|a_{i j}\right\|_{\infty} \leq c$,

(ii) the support of $a_{i j}$ is contained in $\overline{I_{i j}}$,

(iii) for every integer $\ell, 0 \leq \ell \leq k$,

$$
\int x^{\ell} a_{i j}(x) d x=0, \text { and }
$$

(iv) $f(x)=\sum_{-\infty}^{\infty} 2^{i} \sum_{j} a_{i j}(x)$. It is easy to see that for any

$\varphi(x) \in \mathcal{S}$,

(v) $\int f(x) \varphi(x) d x=\sum_{-\infty}^{\infty} 2^{i} \sum_{j} \int a_{i j}(x) \varphi(x) d x$. 
Now, we can prove Theorem (5).

Proof. Let $\varphi(x)$ belong to $\mathcal{S}$ and support of $\varphi(x)$ contained in $[0, \infty)$. By Proposition (14) the functions $a_{i j}(x)$ described in (16) satisfy

$$
\left|a_{i j} * \varphi_{t}(x)\right| \leq c\left(\chi_{\widetilde{I}_{i j}} * h_{t}(x)\right)^{k+2} .
$$

Then, by (v), we get

$$
\left|f * \varphi_{t}(x)\right| \leq c\left(\sum_{i} 2^{i /(k+2)} \sum_{j} \chi_{\widetilde{I}_{i j}} * h_{t}(x)\right)^{k+2} .
$$

If we define

$$
g(x)=\sum_{i} 2^{i /(k+2)} \sum_{j} \chi_{\widetilde{I}_{i j}}(x)
$$

we have

$$
\left|f * \varphi_{t}(x)\right| \leq c\left(g * h_{t}(x)\right)^{k+2},
$$

which proves (6). On the other hand, by Lemma (8), it follows that

$$
\|g\|_{L^{q}(w)} \leq c\left\|\sum_{i} 2^{i /(k+2)} \sum_{j} \chi_{I_{i j}}\right\|_{L^{q}(w)}
$$

Then, taking into account that

$$
\sum_{i} 2^{i / k+2} \chi_{\Omega_{i}}(x) \leq \frac{2^{1 / k+2}}{2^{1 / k+2}-1} f_{-, k+1}^{*}(x)^{1 / k+2},
$$

we obtain the inequality (7).

Application . Let $\lambda>1$ and $r>0$. We define a right hand sided version of the $T_{\lambda, r}$ function of Fefferman and Stein, see [FS], as

$$
T_{\lambda, r, \varphi}^{-} f(x)=\sup _{\delta>0}\left(\delta^{-\lambda} \int_{0}^{\delta} \int_{x-\delta}^{x} t^{\lambda-2}\left|f * \varphi_{t}(y)\right|^{r} d y d t\right)^{1 / r}
$$

where $|\varphi(x)| \leq c /(1+|x|)^{1+\varepsilon}, \varepsilon>0$, and the support of $\varphi(x)$ is contained in $[0, \infty)$. 
Proceeding as in [MW] we obtain that if $\lambda<r$

$$
T_{\lambda, r, \varphi}^{-} f(x) \leq c M^{-}\left(|f|^{r / \lambda}\right)(x)^{\lambda / r}
$$

holds for every $x \in \mathbb{R}$. If $\lambda \geq r$, let $k$ be a non-negative integer such that $\lambda<r(k+2)$. By Theorem (5) we get

$$
\begin{aligned}
T_{\lambda, r, \varphi}^{-} f(x) & \leq c \sup _{\delta>0}\left(\delta^{-\lambda} \int_{0}^{\delta} \int_{x-\delta}^{x} t^{\lambda-2}\left[g * h_{t}(y)\right]^{r(k+2)} d y d t\right)^{1 / r} \\
& =c\left[T_{\lambda, r(k+2), h}^{-} g(x)\right]^{k+2}=c\left[T_{\lambda, s, h}^{-} g(x)\right]^{k+2}
\end{aligned}
$$

with $s=r(k+2)$. Since $\lambda<s$, by (17) with $s$ instead of $r$, we have

$$
T_{\lambda, r, \varphi}^{-} f(x) \leq c\left[M^{-}\left(g^{s / \lambda}\right)(x)\right]^{\lambda / r}
$$

Thus

$$
\begin{aligned}
\int\left[T_{\lambda, r, \varphi}^{-} f(x)\right]^{p} w(x) d x & \leq c \int\left[M^{-}\left(g^{s / \lambda}\right)(x)\right]^{\frac{\lambda p}{r}} w(x) d x \\
& \leq c \int g(x)^{(k+2) p} w(x) d x
\end{aligned}
$$

if $(\lambda p) / r>1$ and $w \in A_{\lambda p / r}^{-}$. Taking into account (7) we obtain the inequality

$$
\int\left[T_{\lambda, r, \varphi} f(x)\right]^{p} w(x) d x \leq c \int f_{-, k+1}^{*}(x)^{p} w(x) d x .
$$

Thus, we have proved the following theorem which generalizes a result of B. Muckenhoupt and R. L. Wheeden [MW].

Theorem 18. Let $p>r / \lambda, k+2>\lambda / r$ and $w \in A_{\lambda p / r}^{-}$. If $\varphi(x) \in \mathcal{S}$ with support contained in $[0, \infty)$, then

$$
\int\left[T_{\lambda, r, \varphi}^{-} f(x)\right]^{p} w(x) d x \leq c \int f_{-, k+1}^{*}(x)^{p} w(x) d x
$$

holds with a finite constant $c$ depending on $\lambda, r, \varphi, w, k$ and $p$. 


\section{REFERENCES}

[FS] C. Fefferman and E. M. Stein, $H^{p}$ spaces of several variables, Acta Math. 129 (1972), 137-193.

[GR] J. García-Cuerva and J. L. Rubio de Francia, Weighted Norm Inequalities and Related Topics, North-Holland, Amsterdam, 1985.

[MW] B. Muckenhoupt and R. L. Wheeden, Norm inequalities for the Littlewood-Paley function $g_{\lambda}^{*}$, Trans. Amer. Math. Soc. 191 (1974), 95-111.

[RS] L. De Rosa And C. Segovia, Weighted $H^{p}$ spaces for one sided maximal functions, Contemporary Mathematics (AMS series) 189 (1995), 161-183.

[S] E. SAWYER, Weighted inequalities for the one sided Hardy-Littlewood maximal functions, Trans. Amer. Math. Soc. 297 (1986), 53-61.

[ST] J. O. Strömberg and A. Torchinsky, Weighted Hardy Spaces, Lecture Notes in Math. Vol. 1381, Springer-Verlag, New York, 1989.

[SW] E. M. Stein And G. Weiss, On the theory of harmonic functions of several variables, I. The theory of $H^{p}$ spaces, Acta Math. 103 (1960), 25-62.

Departmento de Matemáticas

Universidad Nacional de Buenos Aires (UBA)

(1428)-Buenos Aires

República Argentina

CURRENT ADDRESS: Instituto Argentino de Matemática

Saavedra 15,3 er Piso

(1083)-Buenos Aires

República Argentina

EMAIL: segovia@iamba.edu.ar

EMAIL: lderosa@mate.dm.uba.ar

Subject Classification: 1991 Mathematical Subject Classification. Primary 42B30; Secondary 42B25.

KEYWORDS: one-sided weights, one-sided operators, $H^{p}$-spaces

Received: May 11th, 1998. 